\begin{tabular}{l|l} 
REVISTA & $\begin{array}{l}\text { Revista Educación } \\
\text { ISSN: 0379-7082 } \\
\text { ISSN: 2215-2644 } \\
\text { revedu@gmail.com } \\
\text { Universidad de Costa Rica } \\
\text { Costa Rica }\end{array}$
\end{tabular}

\title{
La enseñanza de la filosofía como bien público
}

Cordero R, Joan J.

La enseñanza de la filosofía como bien público

Revista Educación, vol. 43, núm. 2, 2019

Universidad de Costa Rica, Costa Rica

Disponible en: http://www.redalyc.org/articulo.oa?id=44058158006

DOI: https://doi.org/10.15517/revedu.v43i2.31110

Esta obra está bajo una Licencia Creative Commons Atribución-NoComercial-SinDerivar 3.0 Internacional. 


\title{
La enseñanza de la filosofía como bien público
}

The Teaching of Philosophy as a Public Asset

Joan J. Cordero R

Universidad de Costa Rica, Costa Rica

joan.corderoredondo@gmail.com

(iD http://orcid.org/0000-0002-4288-8242

DOI: https://doi.org/10.15517/revedu.v43i2.31110
Redalyc: http://www.redalyc.org/articulo.oa?id=44058158006

Recepción: 31 Octubre 2017

Aprobación: 23 Abril 2019

\begin{abstract}
RESUMEN:
El artículo desarrolla una presentación, a nivel teórico, de una variante del ejercicio práctico de la enseñanza de la filosofía desde una perspectiva de la educación para una ciudadanía global (UNESCO 2011, 2013, 2015, 2016) en tiempos actuales (i.e. educación para la paz y derechos humanos). En el prólogo, la tesis es que la filosofía es un bien público porque su enseñanza potencia el desarrollo estético (social y político), epistemológico (pensamiento ordenado, lógico y consistente) y ético-práctico (democracia, convivencia y civismo) en el alumnado (UNESCO 1972, 2004). En la segunda parte se registra la filosofía como una disciplina que logra proporcionar al individuo una concientización relacional de su dimensión, impacto y situación en el mundo, así como la capacidad de resolver problemas teóricos a nivel práctico y prácticos a nivel teórico, y de dirigir, esforzar y analizar comportamientos y voluntades individuales y colectivas mediante procesos de argumentación y estudio de conocimientos válidos. Se concluye en la tercera parte que, a pesar de la existente situación de crisis, de decaimiento mundial y del poco aprecio por la enseñanza de la filosofía, la disciplina es pertinente para la promoción y ejercicio de prácticas sociales razonables (ciudadanía global), las capacidades cognitivas y conductuales. Las prácticas para la convivencia, el bienestar social y ambiental y la democracia son potenciadas por la filosofía, que desarrolla las capacidades y valores necesarios en cada estudiante para la sustentabilidad de gobernanza (democracia) y el civismo mundial (ciudadanía).
\end{abstract}

Palabras Clave: Enseñanza de la filosofía, bien público, democracia, ciudadanía.

\section{Abstract:}

The goal of this article is to conduct a practical exercise about the teaching of philosophy from a perspective of global citizenship (UNESCO 201 1, 2013, 2015, 2016) (i.e. Peace and Human Rights Education). The first section argues that teaching Philosophy as a subject matter results in enhanced student aesthetics (social and political), epistemology (order, logic and consistence) and ethics (democracy, coexistence and citizenship) (UNESCO 1972, 2004). The second section states that Philosophy as a discipline confers individuals with a better relational awareness of their impact upon the world. They have also reported using argumentation and acquired knowledge in order to improve theoretical problem-solving and strive to analyze individual and collective behavior and will. Students who study Philosophy are more tolerant and understanding of cultural, scientific, social and political phenomena. They also acquire a better attitude towards citizenship and a greater sense of moral action. Lastly, it concludes that despite the global crisis revolving around the lack of interest in the teaching of philosophy, it remains relevant since it fosters in students, cognitive and behavioral skills required for democracy, good governance and global citizenship.

KEYWORDS: Teaching of Philosophy, Public Assets, Citizenship, Democracy.

\section{Prólogo}

\section{1- Del carácter del prólogo}

Como todo prólogo, este expresa lo mismo que será detallado más adelante, prescindiendo, no obstante, de la forma; en tanto preámbulo, dice lo mismo que luego será expresado de otra manera. Para intentar dar énfasis a la dimensión de la filosofía con importancia cívica, el texto trata de constituir una presentación, a nivel teórico, de una variante de su ejercicio práctico: educar para una ciudadanía global (UNESCO, 2011, 2013, 
2015, 2016) en tiempos actuales (i.e. educación para la paz y derechos humanos). La intención de iniciar con un prólogo es un resultado: en tiempos actuales en los que trascurre y se desarrolla la época moderna, específicamente en el modo en que lo hace y tiene suceso el capitalismo (globalidad), habrá que reconocerse que la criticidad no es un a priori estructural ni congénito al ser humano, sino más una construcción y producto de la formación cognitiva del individuo y de la sociabilidad humana. Puesto así, la criticidad es más bien el resultado del arduo y constante trabajo sobre la revisión de los propios postulados y presupuestos de la modernidad filosófica verificados en su pretensión y en el momento de praxis y de ejecución. Desde Kant (1724-1804), la palabra crítica ha connotado límite, alcances; de tal modo que cuando se habla de crítica y de criticidad, se habla desde la tradición y génesis de un determinado pensamiento filosófico que paradigmáticamente inauguró este, al tiempo que se hace referencia a un determinado pensamiento que por saberse limitado es crítico de sí y desde sí (Kant, 1978).

La modernidad filosófica es una temporalidad sociohistórica que, viniendo de estados sociales cuasimonolíticos, avanza sobre su propia complejidad para tornarse diversa y plural. Según esto, la criticidad consiste en la verificación de esto último y en los límites que, en cuanto proceso social e histórico, está sujeto a condiciones contingentes. Se reconoce que la crítica es posible desde la historia; si bien la modernidad en su planteamiento original fue estructurada sobre principios y valores políticos abstractos, en el trascurso del tiempo ha terminado considerando estos valores como fines en sí mismos y no principios regulativos de acción social, la recuperación y reposicionamiento de ello supone la historia y la tradición (Horkheimer, 2000).

\section{2- Para una educación crítica}

La pedagogía crítica reconoce que el proyecto de la construcción de una democracia estable (progreso moral), fornida y progresista todavía no está terminado. El mismo Kant (2013) reconoció que se está en el proceso de la Ilustración, el cual no es un factum, sino un proyecto abierto e inacabado, no es un estado definitivo. Desde este sentido, la pedagogía se presenta como un compromiso con un presente que se está haciendo y supone como necesario conjugar los valores de la razón, libertad y de la igualdad. Crítica significa aquí posibilidad, contingencia, y fomenta la acción humana regulada por el pensar y el pensamiento crítico por el actuar. En este sentido, la educación y la democracia se ocupan a fondo de desarrollar habilidades del pensamiento (sensibilidad estética/cultural y rigurosidad lógica, Terricabras, 1999) en los ciudadanos para la capacidad de emisión de juicios y autogobierno (Lipman 1992; UNESCO 1996; Dewey 1998). En esta medida se favorece la apropiación de lo público (espacio político) y del ejercicio de las prácticas gubernamentales. Lo contrario significaría un retraimiento del individuo hacia sí mismo, un alejamiento de la sociedad (Castilla del Pino, 2001) y de los sistemas políticos.

\section{3- La filosofía como bien público}

En tiempos en los que la teoría de la educación para una ciudadanía y convivencia democrática se avoca en la promoción de los derechos humanos (UNESCO, 2011, 2013, 2015, 2016), el pensamiento complejo y la multi-interdisciplinaridad, así también como en el pensamiento crítico, la enseñanza de la filosofía y puesta en práctica de los tópicos de la filosofía ha venido a constituirse como urgente necesidad y fuente de una educación para la resolución de problemas sociales práxicos basados en la inteligencia personal intercomunicativa y el capital cívico. El reconocimiento de la enseñanza de la filosofía como núcleo del sistema educativo (Lipman, 2010), en tanto momento ético, epistemológico, lógico, estético y antropológico, dentro de la malla curricular ha devenido como fundamento de los horizontes y proyectos de los retos educativos del nuevo milenio (Naciones Unidas, 2015; UNESCO, 2004). 
La filosofía como bien público se relaciona con la práctica cívica mundial. Propone una ampliación constante en el ejercicio de la ciudadanía global y del ámbito público; abandonar las zonas de comodidad que ofrecen la comunidad aislada frente a los problemas y asuntos sociales y públicos-mundiales; propicia la reflexión, la argumentación en virtud de la disponibilidad de la facultad de discernimiento para con el enjuiciamiento; asimismo, trata de propiciar la autonomía, la independencia, y el criterio propio y válido, la participación y contribución en las decisiones de la vida pública cotidiana.

\section{SEGUNDA PARTE}

\section{Introducción}

Un serio diagnóstico de la UNESCO (2011) ${ }^{[1]}$ sobre la situación mundial de la filosofía reveló la carencia, ausencia, decaimiento y debilitamiento del ejercicio de la enseñanza de la filosofía en diversas zonas del mundo. Se ha dado una tendencia global a marginalizar la reflexión filosófica. En la institución escolar, la filosofía ha perdido fuerza y no se ha desarrollado donde ha tenido presencia, y donde la enseñanza de la filosofía no ha tenido presencia ni desarrollo no se atisba su aparición. Al contrario, de manera solapada la filosofía ha venido siendo sustituida por un "conjunto de creencias que tiene por objeto el fomento de un pensamiento (...) mitológico y antirracioanalista” (Vargas, 2012, p. 69), o una específica y única religión y enseñanza moralista doctrinaria (UNESCO, 2011), que en última instancia refiere y evoca a una crisis ${ }^{[2]}$.

La argumentación de fondo hacia la filosofía es que su enseñanza no contribuiría en la formación de ciudadanos en los actuales procesos de globalización y tecnología, ni en la promoción de valores cívicos globales ni en la consecución de capacidades técnico-laborales en el tránsito del siglo XX al XXI ${ }^{[3]}$. No obstante, mientras esta tendencia se cimienta, se reconoce que la enseñanza de la filosofía coadyuva a la creación, promoción y fortalecimiento de sociedades democráticas y plurales, de sociedades intencionadas a la inclusión y caracterizadas por la justicia social global, el reconocimiento de la diversidad cultural y la dignidad humana (derechos humanos) (UNESCO, 2015) en función de una educación que propicie el desarrollo de las capacidades socioemocionales (valores y aptitudes), conductuales (competencias) y cognitivas (conocimientos) (UNESCO, 2013).

La enseñanza de la filosofía potenciaría y fundamentaría en el estudiantado "la libertad de juzgar, cuestionar, discernir, problematizar, conceptualizary argumentar acerca de los graves problemas que afectan a nuestro planeta en general y a nuestro entorno en particular" (Vargas, 2012, p. 67). La enseñanza de la filosofía pretende que cada estudiante trabaje con ideas, contextos y situaciones, lo que acarrea vetas epistemológicas, estéticas, metafísica, éticas y lógicas; también se busca que el alumnado sea ordenado y creativo al pensar, que considere variantes, opciones, versiones, enfoques, perspectivas, mediaciones, que sea curioso, sistemático y reflexivo en la vida individual y conjunta. El valor de la enseñanza de la filosofía se expresa en el estudiantado en la tolerancia y comprensión de los fenómenos culturales, científicos, sociales y políticos, y en la aptitud cívica de estar siendo personas comprometidas con la relación entre conocimiento y actuar moral[4].

El carácter universal de la educación (Savater, 1997) que supone la educación para una ciudadanía global (UNESCO, 2015) es un aspecto, de oficio, filosófico, por el aspecto lógico y estético que implica la autoproducción humana (ontología según Gallardo, 2013 y 2015), por ser una vertiente universalmente humana (abstracto: Todo à parte), y al mismo tiempo por ser situacional y específicamente humano (práctico: Parte à todo). En lo humano, lo universal es la diferencia. Lo común es lo distinto[5]. El respeto hacia y por lo diferentemente cultural, estético y de pensamiento (lo cual refiere a la dimensión ética y axiología) supone un esfuerzo hermenéutico, heurístico y metódico. El ámbito ético y estético supone un esfuerzo cognitivo de entendimiento humano. 
A pesar de la existente situación de crisis, de decaimiento mundial y del poco aprecio por la enseñanza de la filosofía, se reconoce que la disciplina filosófica es pertinente para la promoción y ejercicio de prácticas sociales razonables (democracia y ciudadanía global); también se reconoce que las capacidades cognitivas y conductuales que promociona y tiene por objeto la filosofía son adecuadas para la consecución de los objetivos del nuevo milenio (Naciones Unidas, 2015). Esto se debe a que promociona y conlleva a reflexionar sobre las actuales condiciones de existencia; lo que permite mostrar, por otra parte, que la metodología de la enseñanza de la filosofía tiene un correlato con las situaciones cotidianas y de existencia de los educandos, demuestra que surgen del contexto propio de cada estudiante, de sus intereses y motivaciones.

A este punto, la revisión y actualización curricular se hace fundamental de cara a los efectos y cambios (retos) locales y mundiales que acarrean la revolución científico-técnica del siglo XXI, la intercomunicación, violencia y guerra, desplazamientos humanos, migración y refugiados, cambio climático y desastres ecológicos, desarrollo económico desigual, pobreza y la pobreza extrema. Si estos efectos-cambios suponen un especial tratamiento reflexivo para con el género humano y su hábitat, debido a la autoexigencia de dar cuenta de su propia posición y definición dentro del $\operatorname{cosmos}^{[6]}$, resulta que la filosofía es una de esas disciplinas que permite proporcionar a cualquier persona una concientización relacional de su dimensión, impacto y situación en el mundo, así como la capacidad de resolver problemas teóricos a nivel práctico y prácticos a nivel teórico, y de dirigir, esforzar y analizar comportamientos y voluntades individuales y colectivas mediante procesos de argumentación y estudio de conocimientos válidos y conocimientos válidos y universales ${ }^{[7]}$.

\section{Filosofía como bien público, aspectos contextuales y conceptuales/teóricos}

La discusión sobre las determinaciones teóricas de la enseñanza filosófica ha tenido diferentes frentes y alcances según sea la perspectiva, la concepción que se tenga, la tradición y el paradigma escolar a la que se inscriba (Agratti, 2000; Campiran, 2000; Cerletti, 2000; Lipman, 1992, 2000, 2003, 2010; MEP, 1991, 2015, 2017; Portilla, 2016; Splitter y Sharp, 1996; UNESCO, 2011 [8]). A sabiendas de que dicha determinación teórica acarrea la situación-problema de la definición conceptual, en adelante se entenderá que la filosofía es un producto, una consecuencia lograda de la acción ordenada y sistemática del intelecto humano. Por esta misma razón, la filosofía se entiende también como producto de la acción crítica que hace la inteligencia plástica sobre sí misma, sobre sus límites y posibilidades, a partir de los contextos y modos de relación con la realidad natural e intersubjetiva humana.

Al entender la filosofía como un resultado, es posible distinguir analíticamente la filosofía del filosofar. El filosofar es la fuente que posibilita la filosofía, y esencialmente radica en la capacidad de percibir y sentir, de admirarse y asombrarse de la realidad inmediata en que subyace la existencia del individuo (García, 1983; Hegel, 2000; Terricabras, 1999; Valls, 2001). Al contrario de una versión muy extendida según la cual la filosofía es razón pura y la razón es causa de verdad, la cual se opone a la sensibilidad, se reconoce que la capacidad sensitiva es fuente de admiración y asombro (Gaarder, 1996; García, 1983), lo que a su vez posibilita el filosofar. El reconocimiento de la importancia de la sensibilidad y de la percepción para con la realidad no es despreciable ni ingenua, al contrario, es instancia necesaria y posibilitadora de la filosofía. Desde este sentido, la admiración y la capacidad de asombro es una elemental actitud que viabiliza, en primera instancia, la reflexión y el detenimiento del pensamiento, para que en un segundo momento se dé el pensamiento superior, sistemático y ordenado de la filosofía (Hegel, 2000). Esta pericia indispensable que hace posible a la filosofía es una aptitud y disposición de ánimo que se distingue del método filosófico (García, 1983), método caracterizado por ordenar y mediar lo que resulta inmediatamente captado de la realidad. El filosofar queda anclado en una primera instancia como acción del sujeto frente a la inmediatez del mundo que le aparece $^{[9]}$, y es ante todo una acción/actividad (Fichte, 1982, 1975) con suceso en la existencia misma, una operación interior, intrínseca a la vivencia y sobre los condicionamientos externos y colectivos del sujeto. 
Así como proporcionar una definición de la filosofía sin filosofar no dice nada de la filosofía en su existencia, describir un método filosófico como operación externa o taxativa a la práctica y la sistematización carece de vivacidad y sentido. A este respecto se identifica la pertinencia y necesidad de anclar el filosofar sobre situaciones cotidianas y vivenciales. Para esto, la didáctica en la enseñanza de la filosofía debe ejercerse no en su adaptación básica y tradicional de formato academicista de cursos magistrales centrados en el contenido que el educador presenta, desarrolla y problematiza, sino en una versión alternativa donde "el procedimiento es la investigación dialógica y donde el profesor y alumnado a un tiempo son co-participantes en un aula transformada en comunidad de investigación" (Lipman, 2010, p. 2). En este caso, la filosofía pretende ser una experiencia total de pensamiento en el lenguaje; pretende ser satisfactoria y agradable en sí misma y hacer de la filosofía algo que tiene valor en sí mismo. El supuesto es que el proceso educativo genere actividades de pensamiento entre quienes aprenden. Los altos niveles de cognición en el aula son posibles por la comunidad de investigación, que a su vez supone el cuido en el pensamiento, el diálogo, la escucha atenta y la generación de relaciones entre ideas y razones. El pensamiento y las prácticas para la democracia y la ciudadanía global se relacionan en la medida que se genere y cultive la capacidad de un pensamiento ordenado, contextual y lógico. Se trata de

(...) aprender que tenemos que hacer algo más que explicar lo que decimos y hacemos: tenemos que justificar nuestras acciones y comentarios discutibles. No se trata de exponer la causa que me hizo hacer esto, sino cuál fue el propósito que perseguía al hacerlo. No cómo llegué a mantener esta opinión sino cuales son las razones por las que sigo manteniéndolas. No las condiciones que me empujaron a hacer este juicio, sino los criterios que me guiaron al hacerlo. (Lipman, 2010, p. 7).

La filosofía como bien público también puede ser referenciada como producto de un quehacer filosófico (Gallardo, 1980) dada por la admiración y el asombro, siendo el quehacer filosófico una actividad/exigencia sociohistórica que permite, a quien se asombra, la pregunta como inquietud genérica especulativa. Pero no se trata de un preguntar (se) desinteresado de las condiciones de existencia, sino precisamente sobre estas. Las condiciones de existencia de las sociedades del siglo XXI remiten a los cambios locales y mundiales que acarrean la revolución tecnocientífica, la intercomunicación, globalización, violencia estratégica, exclusión cultural, desarrollo económico desigual, desastres ecológicos y cambio climático (Vargas, 2012; UNESCO, 2015).

Un cuadro sinóptico de la situación mundial actual hace posible la referencia al contexto mundial en tanto actividad/exigencia sociohistórica; de este modo, el quehacer filosófico asume un preguntar interesado, específico, situado y relacional. El tránsito del siglo XX al siglo XXI se caracteriza singularmente por la inherencia de cambio en la manifestación de la cultura y en los modos de vida; es un estadio de permanente y complejos cambios que generan contradicciones sociales, interrelaciones económicas, tipos de dependencia política/jurídica y tensiones diplomáticas. Las rápidas y abruptas transiciones entre matices y modelos tecnológicos, de acceso y uso, por una parte, pero también en cuanto a la transformación social, de interrelación nacional y de interdependencia económica por otra parte, hacen de las condiciones de existencia del siglo XXI una instancia de responsabilidad para los tiempos actuales y para los futuros. Las necesidades del presente exigen un posicionamiento ético racional, crítico/responsable y solidario.

De acuerdo con Vargas (2012), en este cuadro sinóptico se encuentra los temas/eventos del derrumbe de los países socialistas, la agudización de la desigualdad económica-social, las hambrunas, industrialización, tecnología y cambio climático. También están los eventos de la globalización, la ostensión de la tesis del fin de la historia, el choque de civilizaciones, violencia, exclusión y pobreza. Según la UNESCO (2015), en términos educativos los tiempos actuales representan "un nuevo contexto mundial del aprendizaje" (UNESCO, 2015, p.16), esto significa desarrollar las habilidades para una "sociedad de aprendizaje y educación permanente" (UNESCO, 2015, p.14).

En cuanto a la situación global y general en los tiempos actuales, se reconoce la complejidad del fenómeno y las paradojas que arroja. Una de estas es la intensificación de la globalización económica; si bien se ha reducido la pobreza, ha aumentado el subempleo, desempleo juvenil y la precarización laboral. Se sigue presentado 
el fenómeno de la desigualdad entre regiones continentales (el sur global), entre naciones, poblaciones, edades y género. Globalmente los sistemas educativos son desiguales y existen diferentes niveles en la calidad de la educación y los aprendizajes; el estudiantado tiene mayores situaciones ventajosas y propicias para el aprendizaje en los países ricos que en los pobres; de esto se sigue que haya contextos educativos en los cuales el aprendizaje y el conocimiento se da y se logra con mayor naturalidad que en contextos precarios. Otra paradoja es que al tiempo que se da el reconocimiento de la diversidad cultural e histórica de los pueblos y las etnias, se da un incremento espectacular de chauvinismo cultural y religioso, de nacionalismo y el separatismo. Los avances tecnológicos que han facilitado estrechar las grandes distancias culturales y geográficas, también han sido usados para el narcotráfico, y los conocimientos relativos a la seguridad y vigilancia, han sido usados por el terrorismo y para la guerra. Además, "los modelos actuales de crecimiento económico, junto con el crecimiento demográfico y la urbanización, están agotando las reservas naturales no renovables y contaminando el medio ambiente, lo que provoca daños ecológicos irreversibles y el cambio climático" (UNESCO, 2015, p. 16).

Las condiciones de existencia de la sociedad del siglo XXI caracterizadas principalmente por el cambio y los efectos paradoja del avance tecnológico, económico y de interdependencia, acarrean consecuencias para la educación. Al tiempo que se da aparición de un nuevo contexto mundial de aprendizaje, hay 30 millones de infantes privados del derecho a la educación (UNESCO, 2015).

Durante la alta etapa de la tradición escolar centrada en los contenidos y el aprendizaje se logró prever que el proceso tecnológico y el cambio social supondría la modificación paradigmática de la escuela, orientada, en este caso, a generar aprendizajes y logros a lo largo de la vida del escolar. Recuperando del Informe Faure (UNESCO, 1972) las nociones de sociedad de aprendizaje y de educación permanente, el Informe Delors (UNESCO, 1996) propuso una visión integrada de la educación basada en cuatro pilares: aprender a conocer, a hacer, a ser y a vivir juntos, los cuales, a pesar de no ser un plan maestro para la reforma, fue una base para la reflexión de opciones sobre la formulación de políticas públicas educativas. Ambos informes lograron referenciar políticas educativas a nivel mundial, no obstante, para la actualidad presentan límites. El segundo decenio del siglo XXI “representa una nueva coyuntura histórica, pues trae consigo diversos desafíos y nuevas oportunidades para el aprendizaje y el desarrollo humano" (UNESCO 2015, p. 15). Hay una nueva fase histórica "caracterizada por la interconexión y la interdependencia, así como por nuevos niveles de complejidad, inseguridad y tensiones" (UNESCO 2015, p. 15). Si bien estos cambios que vienen acaeciendo tienen consecuencias para la educación, no todos exigen de por sí respuestas a nivel de políticas educativas.

No obstante, lo que resulta un hecho es que estos cambios están creando nuevas condiciones de educación. La educación

(...) no solo requieren prácticas nuevas, sino también nuevos puntos de vista desde los cuales aprehender la naturaleza del aprendizaje y la función del conocimiento y de la educación en el desarrollo humano (...) El nuevo contexto exige que reconsideremos la finalidad de la educación y la organización del aprendizaje (UNESCO, 2015, p. 16).

Las necesidades del presente hacen reconocer la inteligencia humana y el conocimiento como el gran recurso; a diferencia de otro tiempo en el que la materia prima era la generadora de riqueza para las naciones, en la actualidad, la producción y utilización de información y de conocimiento son la instancia posibilitadora del desarrollo y la sustentabilidad. Pero la inteligencia es un fenómeno social; el desarrollo individual es mediado e intermediado por el entorno social; el capital cívico es la inteligencia social humana abocada a la generación de sociedades promotoras de libertad, dignidad y bienestar (derechos humanos). La creatividad, según esto, es la habilidad de resolver conflictos y problemas en relación con la convivencia. Entonces la creatividad también es un actuar novedoso, intencionado y eficiente, que se expresa a nivel del pensar, emprender y organizar, y que tiene como objetivo resolver de la mejor manera posible los graves problemas sociales, económicos y ecológicos. Desde este sentido, la filosofía es ética, y es el conjunto de soluciones para resolver en una instancia práctica la convivencia intersubjetiva y ambiental. 
La filosofía tiene su origen sobre condiciones ambientales sociales e históricas, las cuales a su vez son las propias determinaciones que la hacen viable y situada. Y desarrolla su ejercicio y practicidad concreta a partir de un determinado sitio-problema (sea de conocimiento, político, pedagógico y ambiental), de aquí el carácter interesado, existencial y situacional del preguntarse y de la inquietud genérica especulativa. Un problema puede ser científico (y entonces la inteligencia se ocupará de hacer ciencia), puede ser práctico (y se hará buena técnica), o bien puede ser afectivo, ético, político, artístico, religioso, y en cada caso se enfrentará a los problemas correspondientes, pues "todos los resultados objetivos dependen de una actividad y, por lo tanto, la inteligencia teoría es un caso especial de inteligencia práctica" (Marina, 2010, p. 5). Lo práctico en filosofía refiere a lo ético. La diferencia entre un problema teórico y uno práctico es que el primero se resuelve cuando se tiene la solución, mientras el segundo cuando se ejecuta en práctica la solución. Nótese que no se asume que la finalidad última de la filosofía es alcanzar la verdad, sino utilizar la información disponible para resolver problemas prácticos del comportamiento y vivencia social. Se trata de la situación histórica concreta que posibilita el quehacer filosófico y el cómo atiende los problemas de época, de existencia, de cómo los capta y los asume[10]. Desde este sentido, toda expresión del quehacer filosófico atiende y hace frente a hechos situacionales que son asumidos como problemas por dirimir en la acción social organizada e individual[11].

La filosofía en tanto bien público asume las formas de existencia social como problemas prácticos, asume situaciones fácticas en situaciones problemáticas que generan, suponen y exige, por otra parte, esfuerzo, rigor y sistematicidad $^{[12]}$. Si la filosofía reflexiona sobre sus propias determinaciones y estas son asumidas como exigencias, y si los problemas siempre son una mirada sobre la realidad, específica y determinada de una época, la finalidad de la filosofía es dirigir el buen comportamiento humano colectivo para efectos de resolverlos.

\section{Integridad de la enseñanza de la filosofía en el currículo escolar}

En los debates sobre los presupuestos de la enseñanza de la filosofía se discute sobre la posibilidad y atinencia de impartir, dentro del repertorio escolar, la materia de filosofía en total apego a la tradición del desarrollo del pensamiento y de las ideas, o si se debe, más bien, fomentar la perspectiva de la reflexión filosófica. En el primer caso, en concesión de que la filosofía resultaría radical e inabordable por abstracta, los esfuerzos para introducir la filosofía entre los jóvenes se limitan a buscar formas de hacer más simple y asequible la asignatura. En el segundo caso, se asume la necesidad de cambiar la transmisión de los contenidos del conocimiento filosófico venidos de la tradición y asumir una alternativa que genere actividades de pensamiento entre quienes aprenden (Lipman, 1992) ${ }^{[13]}$. Según esto, enseñar filosofía se ocuparía del recuento histórico del pensamiento filosófico, de sus respectivos problemas y tradiciones; aportaría un valiosísimo andamiaje contextual de los derroteros filosóficos de la admiración e indagación en vistas de la resolución de problemas. Enseñar a filosofar trataría del desarrollo de las cualidades del pensamiento para pensar mejor, de poner en orden los pensamientos y trabajar la expresión y comunicación de estos; enseñar a filosofar se ocupa de

(...) lograr que [el estudiantado] encadenen su pensamiento, que sean consecuentes con lo que afirman, que no olviden los pasos de su propia reflexión, que se centren en una temática dada, que no dejen los temas inconclusos y que fundamenten argumentativamente sus respuestas (Benítez, 2000, p. 30).

No obstante, ambas perspectivas no deben verse escindidas sino yuxtapuestas; en realidad, ambos posicionamientos representan dos manifestaciones de un mismo fenómeno. No es posible introducirse en el tratamiento de los debates y argumentaciones filosóficas en total desconocimiento del contexto que genera una determinada problemática, sus distintas propuestas y perspectivas teóricas en torno a la cual se habilita el debate y se dialoga.

También se discute sobre la integridad de la filosofía como disciplina. Hay quienes se avocan por preservar la integridad de la filosofía como disciplina, esto es, concibiéndola, en la teoría y práctica, como disciplina uniforme, autónoma, consistente en sí misma y sin la necesidad de relación con otras disciplinas. Y hay para 
quienes el valor de la filosofía está en que intensifica la reflexión en todo el currículo; independientemente de la disciplina desde la cual se acometa la enseñanza, se promociona la reflexión, las actividades y el talante filosófico. Estos afirman que estudiantes necesitan que se les animen a ser más reflexivos, detenidos y

(...) críticos en su contacto, con la historia, la política, las matemáticas, el lenguaje. Por ello, valoran los méritos instrumentales de la filosofía, mientras consideran su valor intrínseco, insistiendo en que hay que introducirla y conservarla como asignatura aparte dentro del currículum (Lipman, 1992, pp. 116).

Pero en realidad no es necesario elegir entre enfoques. "Los que han enseñado filosofía a los niños como asignatura específica, se han dado cuenta de que, casi de manera inevitable, incide sobre otras disciplinas" (Lipman, 1992, pp. 116). A estudiantes que se les ha enseñado a ser "sistemáticamente curiosos y naturalmente reflexivos, tienden a extender esa conducta al resto de sus aprendizajes" (Lipman, 1992, pp. 116).

La enseñanza de la filosofía pretende, en última instancia, que el alumnado trabaje con ideas, contextos y situaciones. Si bien la filosofía no es la única disciplina que puede trabajar sobre el pensamiento y las ideas, sí es una disciplina que tiene cierto ahínco en promover la creación de un pensamiento caracterizado por el orden y la consistencia, principalmente por su naturaleza exploratoria, epistemológica y metafísica. En sentido estricto, la filosofía no es una disciplina que trabaje con contenidos curriculares sino sobre los contenidos [14]. También se aspira a que los estudiantes sean ordenados y creativos al pensar, que consideren variantes, opciones, versiones, enfoques, perspectivas, mediaciones, que sean curiosos, sistemáticos y reflexivos en la vida individual y conjunta. No animar al estudiantado a que piense sobre lo que cree y supone sería irresponsable. La intención es producir un cambio global en su conducta, consigo mismo, con el conocimiento, el aprendizaje, la escolaridad y los hábitos que esta supone, con sus compañeros y sus compañeras de aula, la comunidad y con la ciudadanía local y mundial. El valor de la enseñanza de la filosofía tiene expresión en la capacidad de lectura, expresión de sentimientos, opiniones y creencias, de interpretación, de razonamiento, de creatividad y de comportamiento.

La educación filosófica coadyuva a la formación de ciudadanos, promueve el análisis de los eventos, hechos y valores, el pensamiento reflexivo, la criticidad, el juicio fundamentado y la producción de mediaciones cognitivas; promueve el pensamiento complejo y el esfuerzo por la comprensión y entendimiento de un mundo global interrelacionado, plural, diverso y dinámico, en función de evitar el adoctrinamiento, el miedo social y las prácticas totalitarias. La filosofía anima a pensar y hacerse responsable de lo obrado y lo pensado; desde este sentido, el valor de la filosofía radica en ser imprescindible para el buen ejercicio de la ciudadanía y la convivencia conjunta y global.

Una manera de aproximarse al filosofar es efectuando preguntas. Se ha dicho con insistencia que las interrogantes han sido el motor del pensamiento. Pero en cuanto al hecho de formular preguntas, antes que ofrecer una respuesta, vale más el camino abierto ante el ejercicio de la inquietud y la interrogante. En la propuesta constructiva de los fundamentos psicopedagógicos de la educación, una de las nociones más importantes es el desequilibrio cognitivo. Para que el aprendizaje tenga suceso "es necesario crear un desequilibrio cognitivo en los educandos, es decir, obligarlos a cuestionar sus viejas ideas, sus supuestos y presupuestos originales y que las transformen a la luz de nueva evidencia que las cuestiona" (Echeverría, 2004, p. 36).

Atendidas con cierta disposición filosófica, aptitud y detenimiento, las interrogantes mostrarían inquietud y curiosidad, y en segunda instancia verificarían el hecho que estas interrogantes coinciden con las grandes preguntas de la historia del pensamiento filosófico. Lo valioso de las preguntas no estriba sobre el producto de una respuesta última y definitiva. Las explicaciones últimas regularmente entorpecen la reflexión, detienen el pensar atento y el filosofar, precisamente por su carácter pretencioso, esencialista y atemporal. Una explicación suficiente actúa como un final razonable (cierre epistémico tentativo) frente a determinados y 
específicos problemas. Una explicación suficiente considera el contexto y las relaciones en que aparecen las preguntas, al tiempo que permite rectificar y corregir en el camino mismo las respuestas que se van logrando.

Más que la respuesta como resultado último de las preguntas, vale la búsqueda y la complicidad que supone el preguntar dentro del proceso del pensar; desde este sentido, pensar es más una exigencia que una meta. Frente a las preguntas, la intención de buscar razones y explicaciones últimas, precipitan y obstruyen la investigación. Las respuestas actúan como explicaciones frente a singulares argumentos, formulaciones y contextos; el hecho de que las explicaciones tengan un cierre epistémico tentativo no conlleva a afirmar que sea este el final de la investigación. Si un problema es un modo de relación entre un sujeto y un ente o evento, en esto es más apreciable el proceso de indagación como exigencia para el pensamiento que determinar el fin explicativo.

\section{TERCERA PARTE}

\section{Epílogo}

La filosofía se ocupa del pensamiento, de su ordenación y evaluación según principios y reglas. Los alcances se dan desde un espacio intersubjetivo, social; esto es: el diálogo y la comunidad de investigación, el intercambio de perspectivas y de opiniones justificadas con argumentos razonables, que exigen detenimiento, pensar y reflexionar. Esta referencia a la filosofía no pretende resonar exclusivamente lógica; definir la filosofía en cuanto a su aplicación en educación desde un único ámbito, es moverse en contra de su propia práctica; la filosofía es una conjunción de ramas y enfoques que tan solo analíticamente se presentan como partes independientes. En el ejercicio, toda diferenciación ulterior que se hace de la filosofía tiende a diluirse. Los límites son movibles porque una posición ética supone fundamentos epistemológicos, tiene consecuencias o anclajes culturales, o bien porque un posicionamiento ético no se puede fundar sobre nociones incoherentes.

Según Dean (2010), el razonamiento filosófico alienta en el estudiantado la agudización del ingenio y a pensar con disciplina; esta fue la tarea de Descartes (1596-1650), no basta con pensar, hay que pensar con método. Tanto las Reglas para la dirección del espiritu del 1626-8 (Descartes, 2010) como El discurso del método de 1637 (Descartes, 1993) responden a esta idea de forzar el pensamiento a la rigurosidad, al orden y a la investigación. El ejercicio de la ciudadanía tiene que ver con la resolución de problemas y asuntos prácticos/ públicos sobre principios y fundamentos teóricos, sobre las razones y la justificación teoría que acompaña y sustenta lo práctico.

El ejercicio para una ciudadanía global atiende a la práctica del juicio fundamentado y consistente, a la superación de la simple e irresponsable doxa, del dogmatismo y el prejuicio; y se encamina a la construcción de espacios solidarios de argumentación, reflexión, autocorrección y corrección colectiva. El fuerzo intelectual por resultar argumentativamente claro y consistente no implica suponer que en el espacio público y político del civismo, la convivencia y la democracia, exista una última, definitiva y ahistórica verdad. En el espacio público político no hay verdad; su carácter es abierto, agónico y controversial.

La enseñanza de la filosofía posibilita el pensamiento crítico[15] entendido como pensamiento que se apoya en criterios, es autocorrectivo y sensible al contexto (Marina, 2010, p. 5). Por lo primero, es un pensamiento que está basado en razones, especialmente en un tipo de razones basado en criterios o patrones. Por lo segundo, es una forma de investigación, pues toda investigación es autocorrectiva; determina cualquier tipo de error que pueda cometerse a lo largo de la investigación para efectos de corregir. Por lo tercero, se ocupa de aplicar a cada situación particular las reglas apropiadas al caso, y que todos los matices de tales situaciones se tengan en cuenta en su totalidad (Lipman, 2010). El pensamiento crítico está estrechamente relacionado con dar razones, entonces también con hacer preguntas, buscar alternativas y desarrollar la autocorrección y cuido para propiciar el diálogo y la comunidad de investigación. Desde este sentido, la filosofía es epistemología. 
La criticidad radica en el establecimiento de los límites del conocimiento o de los pensamientos. Desde Kant, critica es la determinación de lo posible y de lo no posible en el conocer. Desde esta vertiente, la autocorrección del alumnado en el error tiene una connotación progresiva. Si hay algo, señala Bryan Magee (2000 citado en Dean, 2010), que enseña la filosofía a los niños y a las niñas son dos habilidades fundamentales: "a expresarse con lógica y a ser justo en el debate" (p.46). Debate que tiene origen en la Escuela, instancia que según algunos sociólogos de la educación (Cirigliano, 1972), es la instancia de la reproductividad social, espacio diseñado para preparar a los niños y a las niñas para la vida adulta (Savater, 1997). De allí la manifestación democrática de la vida (Dewey, 1998) escolar en la sociedad y del papel de la Escuela para la construcción de esta (Savater, 1997).

La relación entre ciudadanía global, filosofía y estética en cuanto bien público tiene varios matices; la epistemología aporta desde la lógica la ordenación, coherencia y consistencia en el pensar. Por estética, se tiene la instancia social y política de la emisión del juicio común según su forma (Kant, 2003), lo cual a su vez supone una exigencia cognitiva para efectos de la ética social práctica. El juicio es estético, pero también ético y situacional. Es la ética el conjunto de soluciones para resolver los grandes problemas que afectan al individuo, su individualidad, la instancia intersubjetiva y ambiental. La promoción de la utilización de la información y la cognición para el actuar práctico pasa por la mediación de juzgar adecuadamente. La dirección del comportamiento y la conducta social es la finalidad de la inteligencia, no es, como se supuso en otro momento histórico, alcanzar y tener la verdad. Las prácticas para la convivencia, el bienestar social y ambiental y la democracia son potenciadas por la filosofía, desde este sentido la filosofía es un bien público que desarrolla las capacidades y valores necesarios en el estudiantado para la sustentabilidad de gobernanza y el civismo mundial. La rigurosidad en el pensamiento y la pertinencia lógica son dos de estas habilidades, también lo son la capacidad de comunicación y expresión de lo sentido/pensado por cada estudiante para con los demás. La fundamentación de lo externado, la justificación de las creencias y las razones de las decisiones, en consideración de la contextualización en que aparecen y resultan, son válidos para nuestros pensamientos.

\section{REFERENCIAS}

Agratti, L. (2000). Del método socrático al diálogo como contenido procedimental en la enseñanza. En G. Obiols y E. Rabossi (Comps.), La enseñanza de la filosofía en debate (pp. 185-190). Buenos Aires: Novedades Educativas.

Benítez, L. (2000). Enseñar a filosofar: una reflexión sobre perspectivas y marcos teóricos. En G. Obiols y E. Rabossi (Comps.), La enseñanza de la filosofía en debate (pp. 29-44). Buenos Aires: Novedades Educativas.

Campiran, A. (2000). Didáctica para mejorar la reflexión. Comprensión ordenada del lenguaje. En G. Obiols y E. Rabossi (Comps.), La enseñanza de la filosofía en debate (pp.191-205). Buenos Aires: Novedades Educativas.

Carvajal, Á. (2007). A puntes para una historia de la enseñanza de la filosofía en la educación diversificada costarricense, de 1900 a 1980. Revista electrónica Actualidades investigativas en educación, 7(1), 1-24.

Castilla del Pino, C. (2001). La incomunicación. Barcelona: Península.

Cerletti, A. (2000). Reflexiones para un diagnóstico de aprendizajes filosóficos en la escuela secundaria En G. Obiols y E. Rabossi (Comps.), La enseñanza de la filosofía en debate (pp. 177-184). Buenos Aires: Novedades Educativas.

Cirigliano, G. (1972). Filosofía de la educación. Buenos Aires: HVMANITAS.

Dean, M. (2010). Los niños filósofos de Escocia. Revista Selecciones, 1, 40-46.

Descartes, R. (1993). Discurso del método. (C. Láscaris, Trad.) San José: EDUCA.

Descartes, R. (2010). Reglas para la dirección del espiritu. (J. Navarro, Trad.) Madrid: Alianza.

Dewey, J. (1998). Democracia y educación. España: Morata.

Echeverría, E. (2004). Filosofía para niños. México: SM de Ediciones.

Fichte, J. (1975). Doctrina de la ciencia. Buenos Aires: Aguilar.

Fichte, J. (1982). Reseña de Enesidemo. Madrid: Hiperión. 
Fragomeno, R. (2013, 20 noviembre). La república sin filosofía. Semanario Universidad. Recuperado de http://sema nariouniversidad.com/opinion/la-repblica-sin-filosofa/

Gaarder, J. (1996). Sophie's World. A novel About the History of Philosophy. New York: Berkley Books.

Gallardo, H. (1977). Pensamiento Iberoamericano: las limitaciones de la filosofía clásica. Revista de Filosofía de la Universidad de Costa Rica, 15(40), 109-149.

Gallardo, H. (1980). Del quehacer filosófico. Revista de Filosofía de la Universidad de Costa Rica, 18(47), 61-77.

Gallardo, H. (1984). Teoría y crisis en América Latina. San José: Nueva Década.

Gallardo, H. (2013). Antropologia: la autoproducción bumana. San José: EUNED

Gallardo, H. (2015). América Latina. Producir la torre de Babel. San José: Arlekín.

García, M. (1983). Lecciones Preliminares de Filosofía (6 ed.). México: Editores Mexicanos Unidos.

Hegel, G. W.F. (2000). Enciclopedia de las ciencias filosóficas en compendio. Madrid: Alianza.

Horkheimer, M (2002). Critica de la razón instrumental. Madrid: Editorial Trotta.

Kant, I. (1978). Kritik der reinen Vernunft. Madrid: Alfaguara.

Kant, I. (2003). Kritik der Urteilskraft. Madrid: Machado Libros.

Kant, I. (2013) ¿Qué es la ilustración? Madrid: Alianza.

Lipman, M. (1992). La filosofía en el aula. Madrid: Ediciones de la Torre.

Lipman, M. (1998). Teaching Students to Think Reasonably: Some Finding of the Philosophy for Children Program. The Clearing House, 71(5), 277-280.

Lipman, M. (2000). El descubrimiento de Harry Stottlemeiers. Madrid: Ediciones de La Torre.

Lipman, M. (2003). Thinking in Education. New York: Cambridge University Press.

Lipman, M. (2010). Elfie. Chiapas: Editorial Fray Bartolomé de las Casas, A.C.

Marina, J. (2010). La enseñanza de la filosofía. Conferencia sobre Creación Social. Conferencia llevada a cabo en Creación Social, Madrid.

Ministerio de Educación Pública (MEP). (1991). Programa de estudio de filosofía. Educción diversificada. San José: MEP.

Ministerio de Educación Pública (MEP). (2015). Educar para una nueva ciudadanía: Transformación curricular. San José: MEP.

Ministerio de Educación Pública (MEP). (2017). Programa de estudio de filosofía. Educación diversificada. San José: MEP.

Naciones Unidas. (2015). Objetivos de Desarrollo del Milenio Informe de 2015. Nueva York: Naciones Unidas.

Portilla, M. (2016). Diagnóstico sobre la enseñanza de la filosofía en la educación diversificada. Revista de Filosofía de la Universidad de Costa Rica, 40(143), 177-190.

Savater, F. (1997). El valor de educar. Barcelona: Ariel.

Scheler, M. (1983). El puesto del hombre en el cosmos. Buenos Aires: Losada.

Splitter, L. y Sharp, A. (1996). La otra educación: filosofia para niños y la comunidad de indagación. Ediciones Manantial.

Terricabras, J. (1999). Atrévete a pensar. Barcelona: Paidós.

UNESCO. (1972). Aprender a ser: la educación del futuro: Informe Faure. Paris: UNESCO.

UNESCO. (1984). Teaching and Research in Philosophy: Africa. Studies on teaching and research in philosophy throughout the world, I. Paris: UNESCO.

UNESCO. (1986). Teaching and Research in Philosophy: Asia and the Pacific. Studies on teaching and research in philosophy throughout the world, II. Paris: UNESCO.

UNESCO. (1996). La educación encierra un tesoro: Informe Delors. Paris: UNESCO.

UNESCO. (2009). Teaching Philosophy in American Latina and Caribbean Studies on teaching and research in philosophy throughout the world. Paris: UNESCO. 
UNESCO. (2011). La filosofia: una escuela de la libertad. Enseñanza de la filosofia y aprendizaje del filosofar: la situación actual y las perspectivas para el futuro. París: UNESCO.

UNESCO. (2013). Educación para la ciudadanía mundial. Temas y objetivos de aprendizaje. París: UNESCO.

UNESCO. (2015). Replantear la educación. ¿Hacia un bien común mundial? París: UNESCO.

UNESCO. (2016). Educación para la Ciudadania Mundial. Preparar a los educandos para los retos del siglo XXI. París: UNESCO.

Valls, R. (2001). ¿Qué es la filosofía? Comentario a los \$1-18 de la Enciclopedia de las ciencias filosóficas de Hegel. Areté, 13(2), 141-173.

Vargas, G. (2012) ¿Filosofía para qué?: desafios de la filosofía para el siglo XXI. México D.F: Universidad Autónoma Metropolitana.

\section{Notas}

[1] Aparecido en idioma francés en el 2007 bajo el título La Philosophie, une Ecole de la Liberté-Enseignement de la philosophie et apprentissage du philosopher: état des lieux et regards pour l'avenir se utiliza la traducción oficial de la UNESCO al español aparecida en el 2011 intitulado La filosofia: una escuela de la libertad. Enseñanza de la filosofia y aprendizaje del filosofar: la situación actual y las perspectivas para el futuro. Al respecto puede verse también de la UNESCO los textos sobre la enseñanza e investigación de la filosofía en África (1984), Asia y el Pacifico (1986), y América Latina y el Caribe (2009).

[2] "Cabe evocar al respecto una crisis de la filosofía, pues en la actualidad se tiende hacia una reducción de su enseñanza, un debilitamiento que se debe a varios factores." (UNESCO, 2011, p. 49). Uno de estos es "la frágil condición de los profesores de secundaria y, en particular, la de los profesores de filosofía" (UNESCO, 2011, p. 49), otro es relativo a la formación del profesorado y la poca cantidad de horas impartidas en la disciplina. (UNESCO, 2011).

[3] Sobre el mismo tema, pero en México, puede verse el trabajo de Vargas Lozano intitulado ėncide la filosofía mexicana en la sociedad actual? (Vargas, 2012); en este se verifica la misma intención y sentir al respecto de la filosofía; la tesis, según el subsecretario de Trabajo del sexenio del expresidente mexicano Vicente Fox (2000-2006), es que el sistema no requeriría filósofos sino técnicos. Para el caso costarricense, puede verse lo ejemplificado por Gallardo (1984); en tiempos de los Programas de Ajuste Estructural (PAES), el entonces Ministro de Hacienda en el periodo de gobierno del expresidente Luis Alberto Monge (1982-1986), del Partido Liberación Nacional, Federico Vargas Peralta,

"(...) se refirió a la educación superior (y) habló de prioridades. Defendió el INA (Instituto Nacional de Aprendizaje), al Instituto Tecnológico y a las carreras técnicas de las universidades, pero cuestionó qué es más importante, si preparar filósofos, sociólogos y otros profesionales o instruir en cambio a técnicos que manejen las máquinas que producen la riqueza básica del país" (La Nación, 11/11/1982 citado en Gallardo, 1984, p.11).

Más recientemente puede verse las declaraciones (2013) del entonces precandidato presidencial José Miguel Corrales (Partido Patria Nueva) cuando, ante la pregunta sobre si cerraría la Escuela de Filosofía de la Universidad de Costa Rica, respondió: si se necesita dinero para investigación urgente es mejor cerrar Filosofia y que los muchachos se preparen en ciencias, que es lo que el pais necesita para el desarrollo; para ampliar véase el artículo de Fragomeno (2013) titulado La república sin filosofía.

[4] De este modo, la filosofía se identifica con el dinamismo de la inteligencia artificial humana: conocer, hacer preguntas y actuar. La lógica, la ética, la estética o la introducción a la filosofía correctamente enseñadas podrían proporcionar a los estudiantes valiosos recursos teóricos para pensar en forma consciente, discernir sobre valores; desarrollar una mejor percepción estética del mundo y entender cómo el pensamiento ha coadyuvado con sus concepciones e imaginación al desarrollo de la humanidad. (Vargas, 2012, p. 69).

[5] En Kant (2003 [1790, Kritik der Urteilskraft]), lo humano es el juicio, lo universal/común en su forma es algo particular en su contenido. El juicio, al pretender universalidad hace ingresar al individuo en relación con otros, dándose por enterado que lo universal no es la verdad de su juicio, su contendido, sino la capacidad de enjuiciar, su forma.

[6] Desde el punto de vista filosófico y antropológico, con metodología fenomenológica, puede ver de Max Scheler (1983) el título El puesto del hombre en el cosmos.

[7] Desde esta perspectiva, el filosofar más que una meta es una exigencia. El derecho de la filosofía referido por Jacques Derrida (Marina, 2010), habla del lugar de esta disciplina en el repertorio de los derechos humanos: porque si la filosofía se ocupa de dirigir 
los comportamientos mediante razonamientos, de juzgar adecuadamente la acción mediante argumentos válidos, la reflexión sería el fundamento de los derechos humanos y del ejercicio de la ciudadanía.

[8] En la actualidad el cambio paradigmático curricular se ha dado en el posicionamiento de la educación por habilidades y la resolución de problemas (UNESCO, 2009).

[9] No obstante, ulteriormente este hecho representa, ya de por sí, una mediación con el mundo, aunque sin atisbos de orden y rigurosidad. A este respecto Hegel (2000) menciona que:

"La reflexión aporta en primer lugar una cierta modificación al contenido de la sensación, la intuición, la representación; así, no es más que por medio de una modificación que la verdadera naturaleza del objeto aparece a la conciencia. Así, la reflexión hace aparecer la verdadera naturaleza y este pensamiento es mi actividad, por lo tanto esa naturaleza es también el producto de mi espíritu, en tanto que sujeto pensante..." (p. 278).

[10] Sería, según Gallardo (1980), la apertura de la "conciencia crítica” (p. 62).

[11] En realidad, todo problema, filosófico, psicológico, teórico, práctico... puede vivenciarse colectiva y/o individualmente, es decir, puede comprenderse en el plano social y de psicologismo. Pero en cuanto a la fuente de ello, el problema introduce irrestrictamente la relación entre el sujeto y su contexto, de allí que, en última instancia, aunque un problema sea ubicable en lo particular y expresado en lo individual, ulteriormente conlleva a los modos de relación del individuo con el medio. Se introduce así, la determinación-relación de vivenciarse la existencia con otros individuos.

[12] Haciendo referencia a la expresión actividad filosófica (Gallardo, 1977) se refiere a la actividad teórica como momento o fase de la praxis. Lo cual lleva a que el autor entienda por filosofía clásica el "estudio [de] toda formulación con carácter filosófico que encierre sistemáticamente o tendencialmente la dicotomía entre pensamiento y existencia (teoría-práctica) y/o que se caracterice ideológicamente al sujeto como individuo ahistórico (idealismo, subjetivismo, utilitarismo, historicismo, etc.)” (p. 110).

[13] Sobre los presupuestos de la enseñanza y el ejercicio práctico desde una perspectiva histórica de enfoque curriculista del caso costarricense véase Carvajal (2007).

[14] Existe una clara distinción entre pensar sobre una materia y pensar en una materia (Lipman, 1992).

[15] Según Lipman (1998), el pensamiento crítico es también pensamiento creativo y pensamiento riguroso (caring).

\section{BY-NC-ND}

